\title{
HIGH-RESOLUTION OPTICAL OBSERVATIONS OF EXTREME-ULTRAVIOLET-SELECTED ACTIVE LATE-TYPE STARS
}

\author{
DAMIAN J. Christian \\ Eureka Scientific, Inc., 2452 Delmer Street, Suite 100, Oakland, CA 94602; damian@pha.jhu.edu \\ AND \\ Mihalis MathiOUdAKIS \\ Department of Pure and Applied Physics, Queens University of Belfast, Belfast BT7 1NN, Northern Ireland, UK; M.Mathioudakis@qub.ac.uk \\ Received 2001 November 7; accepted 2002 January 17
}

\begin{abstract}
We present high-resolution optical spectra of EUV-selected late-type stars. We have detected seven new spectroscopic binaries, including a pair of $\mathrm{F}$ stars and an RS Canum Venaticorum candidate. Many of the stars in our sample show remarkable $\mathrm{H} \alpha$ emission with equivalent widths up to $8 \AA$. Several sources show strong He I $5876 \AA$ A emission. We use cross-correlation techniques to estimate line-of-sight rotational velocities and find that they range from less than 5 to over $80 \mathrm{~km} \mathrm{~s}^{-1}$. Several of the stars show a strong Li $6707 \AA$ line, which we use to determine their Li abundances. The EUV-to-bolometric luminosity, a measure of the coronal heating efficiency, is as high as $10^{-3}$. The present sample extends the EUV-selected late-type stars to lower Rossby numbers and makes the coronal saturation boundary better pronounced. Our results indicate that there is a drop in the EUV emission from the corona in late M dwarfs possibly because of a change in the nature of the magnetic dynamo in fully convective stars.
\end{abstract}

Key words: stars: late-type — ultraviolet emission

\section{INTRODUCTION}

Space-borne instrumentation of the late twentieth century has contributed a wealth of information on activity exhibited by late-type stars. This activity is primarily magnetic in nature and is revealed by a range of phenomena including flares and flashes, rotational modulation, or simply the appearance of chromospheric and coronal emission. Surveys of late-type stars reveal that all stars with convective envelopes have a chromosphere (Mathioudakis 1996; Haisch \& Schmitt 1996). Observations with IUE and Einstein have shown that while $\mathrm{G}$ giants have strong X-ray emission, this emission disappears in $\mathrm{K}$ giants. This led to the discovery of the well-defined dividing line in the H-R diagram (Linsky \& Haisch 1979). On the other hand, X-ray emission from the corona is a common property of cool dwarf stars. A ROSAT survey of all late-type dwarfs within $6 \mathrm{pc}$ revealed that $94 \%$ of them had coronae with the nondetections most likely due to insufficient exposure times (Schmitt, Fleming, \& Giampapa 1995). Studies of the X-ray emission from the corona have shown that the most active stars are the fastest rotators (Pallavicini et al. 1981). A picture emerged that this activity is powered by a magnetic dynamo with rotation playing a key role, although the exact mechanism responsible is not quantitatively known.

Rotation is an important aspect of the dynamical behavior of stars; it affects the dynamo action and can modify the mass-loss rate. Stellar rotational velocities depend on a number of parameters, including (1) the angular momentum inherited by the star at the time of its birth, (2) the angular momentum loss through rotational braking, and (3) the rate of angular momentum transport from the stellar interior (Wolff \& Simon 1997). The strength of the dynamo built magnetic field increases with rotation, however, since the dynamo parameters are difficult to determine directly, proxies such as the chromospheric and coronal emission are used. In this context, the Rossby number $R_{o}$, (ratio of rotation period to convective turnover time $\tau_{c}$ ) has often been used instead of the rotational period, when stars with different masses are combined in a single rotation activity relation. This is because the convective turnover time has a very steep dependence on color for F and G stars and is the parameter dominating $R_{o}$, whereas in later spectral types the rotational period dominates because of the weak dependence of $\tau_{c}$ on color (Noyes et al. 1984). In recent years, much observational effort has been concentrated in the study of open clusters (Soderblom 1998). In the younger clusters ( $\alpha$ Per, $50 \mathrm{Myr}$ ), a large number of $\mathrm{G}$ and $\mathrm{K}$ dwarfs are rotating rapidly $\left(v \sin i \geq 100 \mathrm{~km} \mathrm{~s}^{-1}\right)$, whereas in the older Hyades and in the solar neighborhood rapid rotation is confined to the M dwarfs. The Rossby diagram for open clusters has shown that the "plateau" of coronal saturation occurs for $L_{\mathrm{X}} / L_{\mathrm{bol}} \approx 10^{-3}$ to $10^{-4}$. However, the mainsequence solar neighborhood stars surveyed by Mathioudakis et al. (1995b) show no evidence for coronal saturation in the Extreme Ultraviolet Explorer (EUVE) Lexan/B band (58-174 $\AA$ ). This is primarily due to the small number of stars with high rotational velocities. If we were to choose a survey that would be biased toward active stars, an EUV survey would provide such a sample. Soft X-ray and EUV surveys conducted by ROSAT (Pye et al. 1995) and EUVE (Bowyer et al. 1996) were dominated by coronally active, late-type stars. These surveys and their optical follow-up campaigns provided a large sample of new active stars that can offer insights on the mechanisms responsible for the stellar activity.

In this paper, we present high-resolution optical spectra of a sample of active late-type stars (dKe and dMe) selected from these surveys. A brief description of the source selection criteria is presented in $\S 2.1$. In $\S 2.2$, we describe the optical observations, instrumental setup, and data reduction technique. In $\S 3$, we present our results including mea- 
surements of the chromospheric emission in terms of $\mathrm{H} \alpha$ and $\mathrm{H} \beta$, estimates of rotational velocities, and measurements of the $\mathrm{Li}$ I (6707.8 A) line strengths. We derive observed EUV fluxes, the ratio of EUV to bolometric luminosities, and Rossby numbers for the sample, and discuss the sample's activity in $\S 4$. In $\S 5$, we present new spectroscopic binaries and discuss some noteworthy individual sources. Lastly, in $\S 6$ we summarize our results.

\section{OBSERVATIONS AND DATA REDUCTION}

\subsection{Sample Selection}

The objects studied in this paper have been selected from the EUVE survey catalogs and optical identification campaigns. EUVE carried out an all-sky survey using three coaligned telescopes in four bandpasses between 1992 June and 1993 January (Bowyer et al. 1996; Bowyer \& Malina 1991). The four bandpasses were Lexan/B ("Lexan," 58$174 \AA$ ), $\mathrm{Al} / \mathrm{Ti} / \mathrm{C}(156-234 \AA), \mathrm{Ti} / \mathrm{Sb} / \mathrm{Al}$ or "Dagwood" (345-605 A), and $\mathrm{Sn} / \mathrm{SiO}$ or "Tin" (500-740 A). Simultaneously, a fourth telescope, the Deep Survey Instrument (DS), mapped a $2^{\circ} \times 180^{\circ}$ strip of sky along the ecliptic in the two shortest EUV bandpasses, DS Lexan/B (65-190 A) and DS Al/C (167-364 A). The combined effects of higher effective area and longer exposure times resulted in a sensitivity of almost 1 order of magnitude greater than the allsky survey. The resulting EUVE source catalog (Bowyer et al. 1996) contained 734 objects, of which 275 were identified as late-type stars. The EUVE source list was expanded to over 900 sources by comparing marginal EUVE detections within $90^{\prime \prime}$ from the ROSAT PSPC X-ray source list (Lampton et al. 1997). This catalog contributed an additional 81 late-type stars to the EUVE source list. About one-third of the $E U V E$ sources did not have a known optical counterpart in current catalogs, and they were deemed the class of no identification, or NOID. Optical follow-up campaigns of the NOID sources found that the majority of them are coronally active late-type stars (Christian et al. 2001; Mathioudakis et al. 1995a; Craig et al. 1995; 1997; Polomski et al. 1997; Vennes, Korpela, \& Bowyer 1997). It is from these optical campaigns that we have selected the targets for our optical spectroscopy. These sources are at the fainter end of those detected in the EUV surveys, and relatively little is known about their optical properties. Additionally, we have included the two well-known flare stars GJ 182 (dM0e) and GJ 551 (Prox Cen, dM5.5e) for comparison.

\subsection{Optical Observations}

We obtained echelle spectra using the Shane $3 \mathrm{~m}$ telescope at Lick Observatory, the Mayall $4 \mathrm{~m}$ telescope at the Kitt Peak National Observatory (KPNO), and the Anglo-Australian Telescope (AAT) at the Anglo-Australian Observatory (AAO). Data from all three observing campaigns were reduced using standard routines within IRAF.

The Lick observations were conducted on 1999 September 21 and 1999 December 29 with the Hamilton echelle spectrograph (Vogt 1987) and the $2 \mathrm{~K} \times 2 \mathrm{~K}$ thinned CCD (dewar No. 6). This setup covered from 3300 to $9000 \AA$ with spectral resolution of $0.033 \AA$ pixel $^{-1}$ at Ca II and $0.054 \AA$ pixel $^{-1}$ at $\mathrm{H} \alpha$.

The KPNO observations were taken on 2000 October 12 13 with the $4 \mathrm{~m}$ Mayall Telescope and echelle spectrograph. The first night was lost to bad weather and the second night shortened by $25 \%$. We used the echelle spectrograph with red long camera No. 5 and the T2KB CCD (Pilachowski, Willmarth, \& Goad 1982). This setup covered $\approx 4300-7700$ $\AA$, with a resolution of $0.074 \AA \mathrm{pixel}^{-1}$ at $\mathrm{H} \alpha$.

The AAT observations were taken between 2001 June 3 and 5 using the University College London Echelle Spectrograph and the MITLL $2 \mathrm{~K} \times 4 \mathrm{~K} C \mathrm{CD}$ as the detector. The wavelength coverage was $\approx 4700-8200 \AA$, with a resolution of $0.07 \AA$ pixel $^{-1}$ (Diego et al. 1990). The optical observation log for our sample is presented in Table 1 .

In all cases, the wavelength scale was established with ThAr lamps, while flat-field corrections were made using quartz lamps. Several radial velocity standards (e.g., HD 66141,203638 , and 154417) were used to establish the accuracy of the velocity scale, while HR 1544, Feige 15, and LTT 7379 were used for flux calibration. We also obtained optical spectra of GJ 33, 551, 628, 631, 643, 664, 825, 820B, and $860 \mathrm{~A}$, which were used as templates.

\section{OPTICAL EMISSION}

\subsection{Chromospheric Activity Indicators}

Previous optical studies of late-type stars selected from EUV and soft X-ray surveys detections have shown strong chromospheric emission lines. These include follow-up studies of ROSAT WFC (e.g., Fleming, Schmitt, \& Giampapa 1995; Jeffries \& Jewell 1993) and EUVE sources (Craig et al. 1995, 1997; Polomski et al. 1997; Christian et al. 2001). Our late-type star sample shows strong Balmer and He I emission lines. Approximately half of the sources have a Li I 6707.8 A absorption line. We show a sample of sources with both strong $\mathrm{H} \alpha$ and $\mathrm{H} \beta$ in emission in Figure 1 and representative sources with strong $\mathrm{H} \alpha$ emission in Figure 2. $\mathrm{H} \alpha$ equivalent widths (EW) range from $0.2 \AA$ for the less active stars to almost $8 \mathrm{~A}$ for the most active. Five of the EUVEselected stars have $\mathrm{H} \alpha$ EWs $\geq 4 \AA$. Emission-line fluxes and EWs are presented in Table 2. Spectra of binary stars are discussed in $\S 4$.

We present sources with strong lithium I $(6707.8 \AA)$ absorption in Figure 3. The EWs of the Li I (6707.8 $\AA$ ) feature range from a few tens of milliangstroms for stars like EUVE J1145-55.3A to over $600 \mathrm{~m} \AA$ for EUVE J1131-34.6. Lithium abundances have been determined from the observed EWs and the curves of growth of Pavlenko et al. (1995). The $\mathrm{Li}$ abundances and EWs are also listed in Table 2. About $30 \%$ of the sample showed helium lines at 4686, 5876, and $6678 \AA$ ( $\mathrm{He}$ I). Strong lines were detected from EUVE J0419+21.7, GJ 431, EUVE J1811-78.9, EUVE J1931-21.5, and EUVE J2244-33.2A. Sample helium (He I $5876 \AA$ A) emission-line spectra are shown in Figure 4.

\subsection{Rotational Velocities}

We derive projected equatorial velocities $\left(v_{\text {eq }} \sin i\right)$ by cross-correlating the spectra of our sources with computed rotation profiles of low-activity stars of similar spectral type (see $\S 2.2$ ). The spectra of the template sources were convolved with a limb-darkened rotational broadened function (Gray 1992) and then cross-correlated with the unbroadened profiles. Six portions of the spectrum with narrow absorption lines that are free of blends were selected in the wavelength region $6300-6720 \AA$. The width of the cross-correlation function is estimated by fitting a Gaussian. The 
TABLE 1

OBSERVATION LOG

\begin{tabular}{|c|c|c|c|c|c|c|c|c|}
\hline Name & R.A.opt & Decl. $_{\text {opt }}$ & Observatory & Observation Date & $\begin{array}{l}\text { Exposure } \\
\text { (s) }\end{array}$ & $m_{v}$ & Sp. Type & Comment $^{\mathrm{a}}$ \\
\hline RE J0137+ 18AB .............. & 013739.4 & +183533 & KPNO & 2000 Oct 13 & $\begin{array}{r}1800 \\
785\end{array}$ & $\begin{array}{l}9.9 \\
1.08\end{array}$ & $\mathrm{~K} 2$ & J95 \\
\hline EUVE J0318 + 18.4 …....... & 031817.4 & +182420 & $\begin{array}{l}\text { KPNO } \\
\text { Lick }\end{array}$ & $\begin{array}{l}2000 \text { Oct } 13 \\
1999 \text { Sep } 21\end{array}$ & $\begin{array}{l}3600 \\
3000\end{array}$ & 11.8 & $\mathrm{dK} 5-7 \mathrm{e}$ & C95 \\
\hline EUVE J0330 + $18.8 \ldots \ldots \ldots .$. & 033056.1 & +184758 & Lick & $1999 \operatorname{Dec} 29$ & $600(\times 3)$ & 7.8 & F8 & \\
\hline 2RE J0333 + 46A …............ & 033313.5 & +461526 & KPNO & 2000 Oct 13 & 300 & 8.5 & G5 V/IV & J95 \\
\hline EUVE J0419 + $21.7 \ldots \ldots \ldots \ldots$ & 041938.5 & +214614 & $\begin{array}{l}\text { KPNO } \\
\text { Lick }\end{array}$ & $\begin{array}{l}2000 \text { Oct } 13 \\
1999 \text { Sep } 21\end{array}$ & $\begin{array}{l}1800(\times 3) \\
1800\end{array}$ & 11.7 & $\mathrm{dM} 2 \mathrm{e}$ & C95 \\
\hline GJ $182 \ldots \ldots \ldots \ldots \ldots \ldots \ldots$ & 045934.8 & +014701 & KPNO & 2000 Oct 13 & $\begin{array}{l}600(\times 2) \\
900 \\
900\end{array}$ & 10.1 & $\mathrm{dM} 0$ & \\
\hline EUVE J0702-01.8........... & 070259 & -014839 & Lick & 1999 Dec 29 & 1800 & 14.9 & $\mathrm{dM} 2 \mathrm{e}$ & $\mathrm{CC} 97$ \\
\hline RE J0723 + $20 \ldots \ldots \ldots \ldots$ & 072344 & +202506 & KPNO & 2000 Oct 13 & 600 & 9.9 & $\mathrm{dK} 5 \mathrm{e}$ & J95 \\
\hline EUVE J0825-16.3 ............ & 082552 & -162246 & AAT & $\begin{array}{l}\text { 2001 Jun } 3 \\
\text { 2001 Jun } 5\end{array}$ & $\begin{array}{l}1800 \\
1200\end{array}$ & 10.8 & $\mathrm{dM} 0 \mathrm{e}$ & CC97 \\
\hline $\begin{array}{l}\text { EUVE J0831 + 40.2A ....... } \\
\text { EUVE J0831 + 40.2B ...... }\end{array}$ & $\begin{array}{l}083102.0 \\
083101.8\end{array}$ & $\begin{array}{l}+401210 \\
+401212\end{array}$ & $\begin{array}{l}\text { Lick } \\
\text { Lick }\end{array}$ & $\begin{array}{l}1999 \operatorname{Dec} 29 \\
1999 \operatorname{Dec} 29\end{array}$ & $\begin{array}{l}3600 \\
3600\end{array}$ & $\begin{array}{r}13.3 \\
\ldots\end{array}$ & $\begin{array}{r}\mathrm{dM} 4 \mathrm{e} \\
\ldots\end{array}$ & $\mathrm{C} 01$ \\
\hline 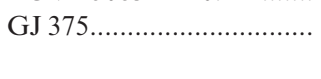 & 095834.32 & -462530 & AAT & $\begin{array}{l}2001 \text { Jun } 4 \\
2001 \text { Jun } 5\end{array}$ & $\begin{array}{l}1800 \\
1800\end{array}$ & 11.3 & $\mathrm{dM} 3.5 \mathrm{e}$ & \\
\hline EUVE J1004 + $50.3 \ldots \ldots \ldots .$. & 100421.7 & +502317 & Lick & 1999 Dec 29 & 3600 & 11.3 & $\mathrm{dM} 3 \mathrm{e}$ & P97 \\
\hline EUVE J1131-34.6........... & 113155.4 & -343627 & AAT & 2001 Jun 3 & $1800(\times 3)$ & 11.5 & $\mathrm{dM} 2.5 \mathrm{e}$ & P97 \\
\hline 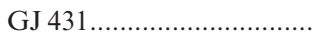 & 113146.5 & -410247 & AAT & 2001 Jun 4 & 1800 & $12.9^{\mathrm{b}}$ & $\mathrm{dM} 3.5$ & \\
\hline EUVE J1145-55.3A ......... & 114552.0 & -552046 & AAT & 2001 Jun 3 & 1800 & 10.4 & $\mathrm{~K}$ & P97 \\
\hline EUVE J1258-70.4 ........... & 125824.0 & -702906 & AAT & 2001 Jun 4 & $\begin{array}{l}1800 \\
1500(\times 2)\end{array}$ & $\ldots$ & $\mathrm{Ge}$ & B96 \\
\hline & & & & 2001 Jun 5 & 1800 & & & \\
\hline EUVE J1305-40.9 …....... & 130530 & -405625 & AAT & 2001 Jun 4 & 1800 & 13.3 & dMle & CC97 \\
\hline GJ $551 \ldots \ldots \ldots \ldots \ldots \ldots \ldots$ & 142942.9 & -624046 & AAT & 2001 Jun 4 & 1500 & 11.05 & $\mathrm{dM} 5.5 \mathrm{e}$ & Prox Cen \\
\hline EUVE J1501-43.6........... & 150116 & -433932 & AAT & $\begin{array}{l}2001 \text { Jun } 4 \\
\text { 2001 Jun } 5\end{array}$ & $\begin{array}{l}1800 \\
1200(\times 2)\end{array}$ & 11.9 & $\mathrm{dM}: \mathrm{e}$ & L97 \\
\hline EUVE J1521 + $20.9 \ldots \ldots \ldots$. & 152153 & +205833 & AAT & 2001 Jun 3 & 720 & 10.1 & $\mathrm{dM} 0 \mathrm{e}$ & \\
\hline EUVE J1713-85.8 …........ & 171308.5 & -855209 & AAT & 2001 Jun 3 & 1800 & 11.7 & $\mathrm{dK} 5 \mathrm{e}$ & P97 \\
\hline EUVE J1716-26.5 ............ & 171613.4 & -263246 & AAT & 2001 Jun 3 & $60(\times 5)$ & 6.3 & $\mathrm{dK} 5$ & B96 \\
\hline EUVE J1811-78.9 ........... & 181114.7 & -785929 & AAT & 2001 Jun 3 & 1800 & 12.4 & $\mathrm{dM} 5.5 \mathrm{e}$ & P97 \\
\hline EUVE J1931-21.5 ........... & 193126 & -313416 & AAT & 2001 Jun 3 & 1800 & 12.3 & dM5e & $\mathrm{CC} 97$ \\
\hline EUVE J2009-23.2 ........... & 200923 & -231311 & AAT & $\begin{array}{l}\text { 2001 Jun } 4 \\
2001 \text { Jun } 5\end{array}$ & $\begin{array}{l}1500 \\
1500\end{array}$ & 11.4 & $\mathrm{~K} 4$ & L97 \\
\hline EUVE J2056-17.1 ........... & 205602 & -171048 & $\begin{array}{l}\text { KPNO } \\
\text { AAT }\end{array}$ & $\begin{array}{l}2000 \text { Oct } 13 \\
2001 \text { Jun } 3 \\
2001 \text { Jun } 4 \\
2001 \text { Jun } 5 \\
\text { 1999 Sep } 21\end{array}$ & $\begin{array}{l}1200 \\
300(\times 20) \\
200(\times 16) \\
200(\times 33) \\
600(\times 2)\end{array}$ & 10.4 & $\mathrm{dM} 0 \mathrm{e}$ & M95b \\
\hline EUVE J2110-19.3 ........... & 211005.3 & -191956 & AAT & 2001 Jun 5 & 955 & 11.8 & $\mathrm{dM} 5 \mathrm{e}$ & P97 \\
\hline EUVE J2113 + $04.2 \ldots \ldots \ldots .$. & 211334.8 & +041406 & AAT & 2001 Jun 5 & 900 & 10.7 & $\mathrm{~K} 4$ & L97 \\
\hline EUVE J2141-14.0 ........... & 214132.9 & -140251 & AAT & 2001 Jun 3 & $120(\times 3)$ & 5.2 & $\mathrm{G} 2 \mathrm{~V}$ & \\
\hline EUVE J2223 + $25.3 \ldots \ldots \ldots .$. & 222355.6 & +252335 & Lick & 1999 Sep 21 & 3600 & 12.2 & $\mathrm{dK} 7 \mathrm{e}$ & $\mathrm{C} 01$ \\
\hline EUVE J2233-09.6............ & 223322.4 & -093653 & Lick & $\begin{array}{l}\text { 1999 Sep } 21 \\
2001 \text { Jun } 5\end{array}$ & $\begin{array}{l}3600 \\
1500\end{array}$ & 12.9 & $\mathrm{dM} 3.5 \mathrm{e}$ & $\mathrm{C} 95$ \\
\hline RE J2244 + 17A ................ & 224441.6 & +175418 & AAT & 2001 Jun 5 & 600 & 8.8 & K0 & J95 \\
\hline EUVE J2244-33.2A ......... & 224457.7 & -331459 & AAT & 2001 Jun 5 & 1800 & 12.1 & dM5e & P97 \\
\hline EUVE J2244-33.2B ........ & 224459.8 & -331524 & AAT & 2001 Jun 5 & 1500 & 13.3 & $\mathrm{dM} 5.5 \mathrm{e}$ & P97 \\
\hline EUVE J $2329+41.4 \mathrm{~B} \ldots \ldots .$. & 232923.2 & +412806 & Lick & 1999 Sep 21 & 2400 & 11.9 & $\mathrm{dM} 4.5 \mathrm{e}$ & C95 \\
\hline EUVE J2332-01.2........... & 233215.6 & -011819 & AAT & 2001 Jun 3 & 720 & 14.5 & dMle & V97 \\
\hline
\end{tabular}

Noте.--Units of right ascension are hours, minutes, and seconds, and units of declination are degrees, arcminutes, and arcseconds.

a (B96) Bowyer et al. 1996 (2nd EUVE Catalog), (C01) Christian et al. 2001, (C95) Craig et al. 1995, (CC97) Craig et al. 1997, (J95) Jeffries, Bertram, \& Spurgeon 1995, (M95b) Mathioudakis et al. 1995a, (P97) Polomski et al. 1997, and (V97) Vennes et al. 1997.

b $B$ magnitude.

value of the slowest rotation that can be detected depends on both the spectral resolution and signal-to-noise ratio. Errors in our analysis are typically in the range of $2-7 \mathrm{~km}$ $\mathrm{s}^{-1}$. They are estimated by comparing the rotational velocities derived from different wavelength regions, as well as from multiple spectra of the same source. In Table 3, we list the rotational velocities of the sources.

The $v_{\text {eq }} \sin i$ values were used to derive rotational periods. We do not expect the sin $i$ dependence to introduce any difficulties in this analysis. Queloz et al. (1998) have constructed 


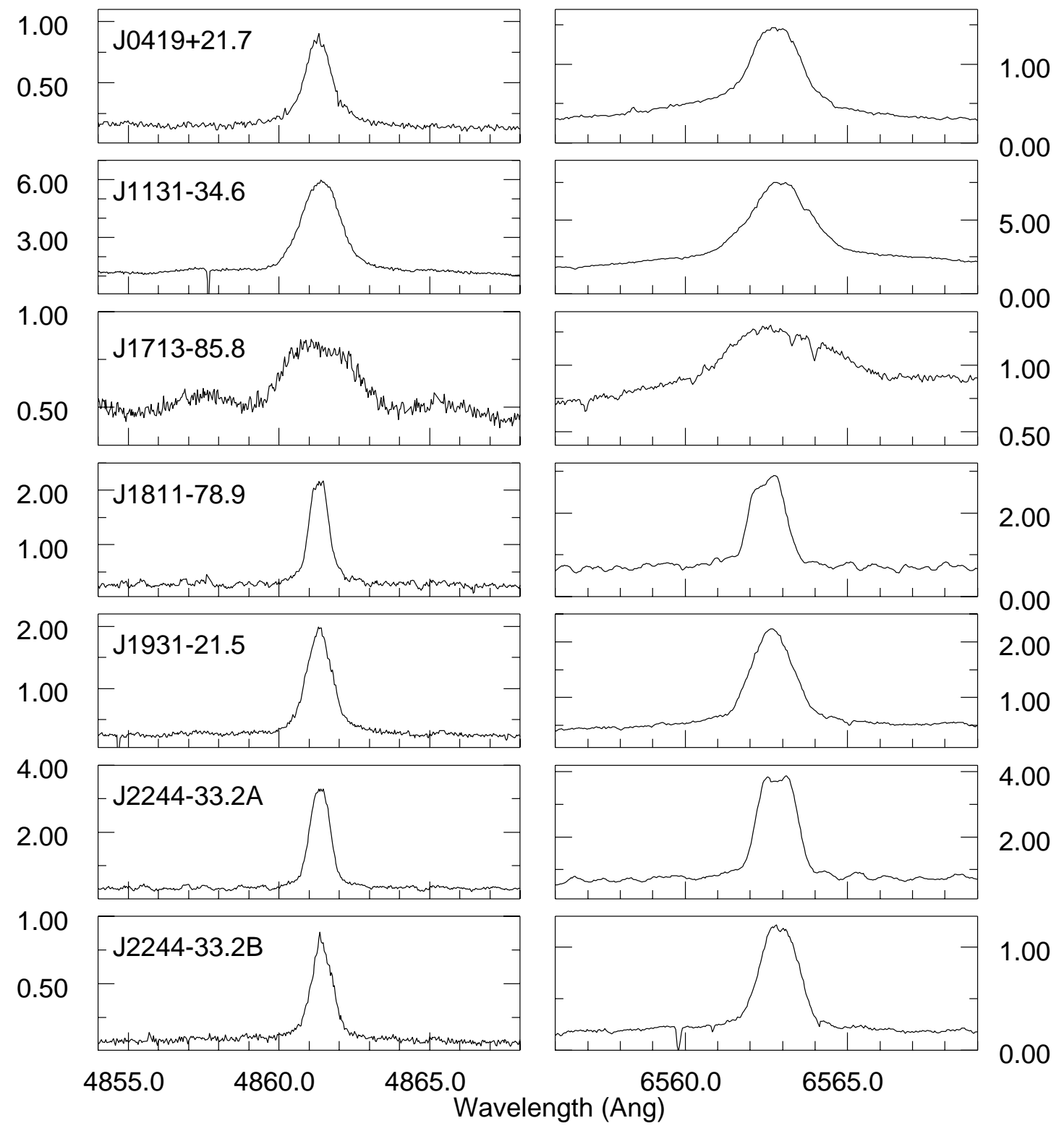

FIG. 1.- Representative sources with strong $\mathrm{H} \alpha$ and $\mathrm{H} \beta$ emission. $\mathrm{H} \beta$ is shown on the left and $\mathrm{H} \alpha$ on the right. Ordinate is flux in units of $10^{-13}$ ergs $\mathrm{cm}^{-2}$ $\mathrm{s}^{-1} \AA^{-1}$.

the cumulative distributions of $\log \sin i$ and find a mean value of $\log \sin i=-0.13$ and a $90 \%$ probability for $\log \sin i>-0.38$. Rossby numbers for the stars in our sample were determined from the rotation periods and the convective overturn times of Noyes et al. (1984). The $\sin i$ statistics introduce a small scatter in the Rossby diagram. The main source of scatter is introduced by the range of activity levels ( $\left.L_{\mathrm{EUV}} / L_{\mathrm{bol}}\right)$ at a given Rossby number.

\section{CORONA ACTIVITY}

\subsection{EUV Fluxes and $L_{\mathrm{EUV}} / L_{\mathrm{bol}}$}

In order to derive the EUV luminosities for our sample, we must convert the observed count rates into fluxes. This calculation requires a coronal emissivity model and an estimate of the interstellar hydrogen column density. We calculated the observed fluxes using the coronal model of Monsignori-Fossi \& Landini (1994) for $\log T=6.8$. The neutral hydrogen column $N(\mathrm{H})$ densities along the line of sight were derived using the three-dimensional interpolation method of P. Jelinsky (1996, private communication). ${ }^{1}$ This model uses a large database of hydrogen column densities (Fruscione et al. 1994) from EUV, UV, and ground-based measurements of individual stars. In general, the dependence of the fluxes on column density is small $(50 \%$ changes in $N(\mathrm{H})$ result in flux changes of $\leq 25 \%$ ), because these objects are nearby and located toward low ISM column densities. We then used bolometric magnitudes and the observed Lexan/B fluxes to calculate the ratio of EUV to bolometric luminosity $\left(L_{\mathrm{EUV}} / L_{\mathrm{bol}}\right)$, which is a measure of the coronal heating efficiency.

The extensive studies of late-type stars over the past years have revealed that the ratio of X-ray-to-bolometric luminosities $L_{\mathrm{X}} / L_{\mathrm{bol}}$ spans in the range of $\sim 10^{-6}$ to $10^{-3}$

\footnotetext{
${ }^{1}$ See http://archive.stsci.edu/euve/ism/ismform.html
} 


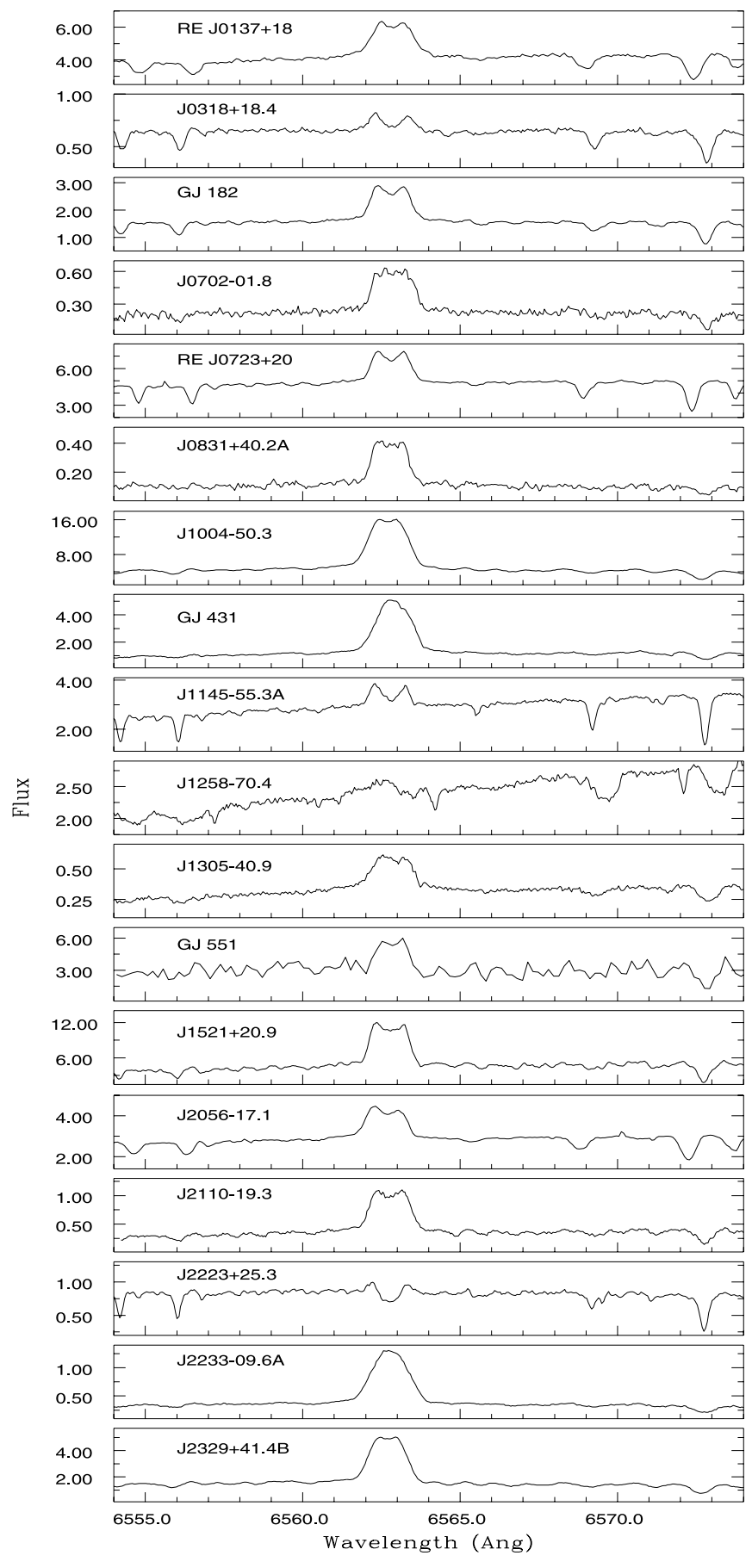

FIG. 2.- Representative spectra of sources with strong $\mathrm{H} \alpha$ emission. Ordinate is the same as Fig. 1.

(Agrawal, Rao, \& Sreekantan 1986; Fleming et al. 1995). Jeffries \& Jewell (1993) found a similar range for the ratio of $L_{\mathrm{EUV}} / L_{\mathrm{bol}}$ for a sample of late-type stars observed with the ROSAT WFC. In Figure 5, we plot the $L_{\mathrm{EUV}} / L_{\mathrm{bol}}$ as a function of the $B-V$ color. We have included the $\mathrm{K}$ and $\mathrm{M}$ dwarfs from the Bowyer et al. (1996) and Lampton et al. (1997), the K and M stars from Mathioudakis et al. (1995b), and M stars from the EUVE Right Angle Program (Christian et al. 1999). Similar to previous studies, the K stars are clustering between $L_{\mathrm{EUV}} / L_{\mathrm{bol}} \approx 10^{-6}$ and $10^{-3.5}$, with the $\mathrm{M}$ dwarfs having values as high as $10^{-3}$. James et al. (2000) have suggested that there is an exclusion zone of coronal activity in the $\log \left(L_{\mathrm{X}} / L_{\mathrm{bol}}\right)$ range of -4 to -3.3 . However, we find no such evidence in our sample. The earlier conclusion was most likely based on poor sampling. Our results indicate that there is a drop in the maximum levels of EUV emission for $(B-V)>1.5$ (spectral types later than M4.5).

For our sample, the average value of $\log \left(L_{\mathrm{EUV}} / L_{\mathrm{bol}}\right)$ for $(B-V)<1.5$ at the saturation boundary is -3.3 , while the average value of $\log \left(L_{\mathrm{EUV}} / L_{\mathrm{bol}}\right)$ for $(B-V)>1.5$ is -3.8 . A simple linear fit to the ratio of $\log \left(L_{\mathrm{EUV}} / L_{\mathrm{bol}}\right)$ for $(B-V)<1.5$ finds a weak positive correlation with a coefficient of 0.4 , and fits to the ratio for $(B-V)>1.5$ find a negative correlation with coefficient of -0.7 . Küker \& Rüdiger (1999) have suggested that the mechanism of field generation changes completely at the cool end of the main sequence. The dynamo action of fully convective stars is generated by an $\alpha^{2}$ dynamo dominated by the stellar rotation rate, as opposed to an $\alpha \Omega$ for objects with a radiative core. This small drop that we see in the coronal activity levels of the most active $\mathrm{M}$ dwarfs may indicate this change in the nature of the magnetic dynamo.

\subsection{Rossby Number and Activity}

We show the ratio of $L_{\mathrm{EUV}} / L_{\mathrm{bol}}$ versus Rossby number in Figure 6 . The figure includes the stars of this paper, as well as the sample of Mathioudakis et al. (1995b). With the addition of the new EUV-selected active dwarfs, the saturation boundary in this diagram becomes evident. Saturation is thought to be related with the limited area of the stellar surface. As more magnetic field is generated by increased rotation and deeper convection, a point is reached where the stellar surface is completely covered with active regions and the emission reaches a maximum (Vilhu \& Walter 1987). A comparison of Figure 6 with the Figure $4 b$ of Mathioudakis et al. (1995b) shows that chromospheric saturation occurs at higher Rossby numbers $\left(R_{o}<0\right)$ than coronal saturation $\left(R_{o}<-1\right)$. In other words, more magnetic energy is required to saturate the corona than the chromosphere. However, the study of chromospheric saturation has been limited to individual spectral lines. The atmospheric models of Houdebine et al. (1996) show that as the atmospheric pressure increases, a larger proportion of the magnetic energy is radiated in the form of continuum, while chromospheric lines saturate. The radiative losses of the atmosphere therefore seem to be displaced from the chromosphere, in low-activity stars, to the upper atmosphere in active stars. An examination of EUV spectra of active stars reveals that most of the flux in the EUVE Lexan/B band (58-174 A) is due to continuum emission. Our finding therefore agrees with the model calculations.

At Rossby numbers of $\approx-2$, the level of saturation is known to decline because of the supersaturation effect (Stauffer et al. 1997). This decline may be supported by our observations; however, given the small number of stars at very high rotational velocities, we cannot derive a firm conclusion for the present sample.

James et al. (2000) suggest that this effect may be related to the reduction of the X-ray-emitting volume of late-type stars due to centrifugal stripping. As the increased rotational velocity decreases the surface gravity, the hot coronal plasma will become unstable and cool to chromospheric temperatures. More cool loops emitting in chromospheric lines can exist, but not in X-rays. In addition, if the dynamo itself saturates, the X-ray emission would be expected to 
TABLE 2

Emission-Line Results for EUVE Late-type Stars

\begin{tabular}{|c|c|c|c|c|c|c|c|c|c|c|c|}
\hline \multirow[b]{2}{*}{ SOURCE } & \multicolumn{3}{|c|}{ Line Fluxes $\left(10^{-13} \mathrm{ergs} \mathrm{cm}^{-2} \mathrm{~s}^{-1} \AA^{-1}\right)$} & \multicolumn{3}{|c|}{ EQUIVALENT WidTHS $(\AA)$} & \multirow[b]{2}{*}{ FLUX $_{\text {EUV }}{ }^{b}$} & \multirow{2}{*}{$\begin{array}{c}\mathrm{Li} \\
(\mathrm{m} \AA)\end{array}$} & \multirow[b]{2}{*}{$\log N(\mathrm{Li})^{\mathrm{c}}$} & \multirow[b]{2}{*}{ SP. TYPE } & \multirow[b]{2}{*}{ Comment } \\
\hline & $\mathrm{H} \alpha$ & $\mathrm{H} \beta$ & $\mathrm{He}^{\mathrm{a}}$ & $\mathrm{H} \alpha$ & $\mathrm{H} \beta$ & $\operatorname{He}(5876)$ & & & & & \\
\hline RE J0137+ 18AB ............. & 4.2 & 4.3 & $\ldots$ & 0.77 & 0.43 & $\ldots$ & 9.5 & 466 & 3.0 & $\mathrm{~K} 2$ & \\
\hline EUVE J0318 + 18.4 ........... & 0.20 & $\ldots$ & $\ldots$ & 0.26 & $\ldots$ & $\ldots$ & 0.6 & 208 & 2.0 & $\mathrm{dK} 5-7 \mathrm{e}$ & KPNO \\
\hline EUVE J0330 + $18.8 \ldots \ldots \ldots \ldots$ & $\ldots$ & $\ldots$ & $\ldots$ & $\ldots$ & $\ldots$ & $\ldots$ & 0.6 & 30 & $\ldots$ & F8 & \\
\hline 2RE J0333 + 46A ............. & $\ldots$ & $\ldots$ & $\ldots$ & $\ldots$ & $\ldots$ & $\ldots$ & 7.0 & 219 & 3.0 & G5 V/IV & \\
\hline EUVE J0419+ $21.7(1)^{\mathrm{d}} \ldots .$. & 1.1 & 0.40 & $\ldots$ & 6.5 & 3.0 & 0.2 & 1.4 & $\ldots$ & $\ldots$ & $\mathrm{dM} 2 \mathrm{e}$ & Low \\
\hline EUVE J0419 + $21.7(\mathrm{~h}) \ldots \ldots$. & 1.6 & 0.59 & $\ldots$ & 6.4 & 7.3 & 0.4 & $\ldots$ & $\ldots$ & $\ldots$ & & High \\
\hline EUVE J0419 + 21.7 (f) ....... & 2.8 & 0.81 & $\ldots$ & 9.3 & 5.1 & 0.5 & ... & $\ldots$ & $\ldots$ & & Flare \\
\hline GJ $182 \ldots \ldots \ldots \ldots \ldots \ldots \ldots \ldots \ldots$ & 7.6 & 3.3 & 0.4 & 1.4 & 1.2 & 0.09 & 11.6 & 263 & 1.5 & $\mathrm{dM} 0 \mathrm{e}$ & \\
\hline EUVE J0702-01.8 ........... & 0.56 & 0.13 & $\ldots$ & 2.7 & 3.1 & $\ldots$ & 16.7 & $\ldots$ & $\ldots$ & $\mathrm{dM} 2 \mathrm{e}$ & \\
\hline RE J0723 + $20 \ldots \ldots \ldots \ldots \ldots \ldots$ & 3.3 & 2.3 & $\ldots$ & 0.7 & 0.8 & $\ldots$ & 7.4 & $<20$ & $\ldots$ & $\mathrm{dK} 5 \mathrm{e}$ & \\
\hline EUVE J0825-16.3 (b) ${ }^{\mathrm{e}} \ldots .$. & 2.3 & 1.9 & $\ldots$ & 0.6 & 0.6 & $\ldots$ & 9.1 & $\ldots$ & $\ldots$ & dM0e & Binary \\
\hline EUVE J0825-16.3 (r) ....... & 1.8 & 1.6 & $\ldots$ & 0.5 & 0.4 & $\ldots$ & $\ldots$ & $\ldots$ & $\ldots$ & $\mathrm{dM} 0 \mathrm{e}$ & Binary \\
\hline EUVE J0831 + 40.2A ....... & 0.4 & 0.17 & $\ldots$ & 3.8 & 3.0 & $\ldots$ & 6.5 & $\ldots$ & $\ldots$ & $\mathrm{dM} 4 \mathrm{e}$ & \\
\hline EUVE J1004 + $50.3 \ldots \ldots \ldots \ldots$ & 17.8 & 5.0 & $\ldots$ & 4.0 & 3.1 & 0.6 & 5.2 & $\ldots$ & $\ldots$ & $\mathrm{dM} 3 \mathrm{e}$ & \\
\hline EUVE J1131-34.5 ........... & 15.0 & 7.4 & 0.8 & 7.8 & 5.6 & 0.5 & 6.4 & 611 & 2.2 & $\mathrm{dM} 2.5 \mathrm{e}$ & \\
\hline EUVE J1145-55.3A ......... & 0.6 & $\ldots$ & $\ldots$ & 0.20 & $\ldots$ & $\ldots$ & 5.6 & 181 & 0.4 & $\mathrm{Ke}$ & \\
\hline EUVE J1258-70.4 „........... & 0.53 & $\ldots$ & $\ldots$ & 0.2 & $\ldots$ & $\ldots$ & 1.6 & 414 & $\ldots$ & $\mathrm{Ge}$ & \\
\hline GJ 375 (b) ........................... & 2.6 & 1.6 & 0.1 & 2.2 & 3.7 & 0.2 & 8.0 & $\ldots$ & $\ldots$ & $\mathrm{dM} 3.5 \mathrm{e}$ & Binary \\
\hline 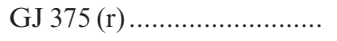 & 2.7 & 2.0 & 0.2 & 2.3 & 4.7 & 0.4 & $\ldots$ & $\ldots$ & $\ldots$ & & \\
\hline 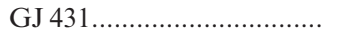 & 5.4 & 3.1 & 0.2 & 4.8 & 5.3 & 0.3 & 6.0 & $\ldots$ & $\ldots$ & $\mathrm{dM} 3.5 \mathrm{e}$ & \\
\hline EUVE J1305-40.9 ........... & 0.55 & 0.30 & $\ldots$ & 1.9 & 1.0 & $\ldots$ & 9.6 & 450 & 2.0 & dMle & \\
\hline 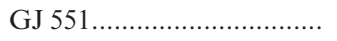 & 4.6 & 1.7 & $\ldots$ & 2.0 & 2.3 & $\ldots$ & 10.3 & $<40$ & $\ldots$ & $\mathrm{dM} 5.5 \mathrm{e}$ & \\
\hline EUVE J1501-43 (b) ......... & 4.3 & 2.1 & 0.2 & 1.8 & 1.7 & 0.2 & 10.7 & $<20$ & $\ldots$ & $\mathrm{dM}: \mathrm{e}$ & Binary \\
\hline EUVE J1501 - 43 (r) .......... & 5.3 & 3.2 & 0.2 & 2.2 & 2.7 & 0.2 & $\ldots$ & $\ldots$ & $\ldots$ & & Binary \\
\hline EUVE J1521 + $20.9 \ldots \ldots \ldots \ldots$ & 10.2 & 8.0 & 0.7 & 2.2 & 2.6 & 0.2 & 13.0 & $<40$ & $\ldots$ & dM0e & \\
\hline EUVE J1713-85.8 ............ & 1.5 & 0.94 & $\ldots$ & 1.7 & 1.9 & $\ldots$ & 5.6 & $\ldots$ & $\ldots$ & $\mathrm{dK} 5 \mathrm{e}$ & \\
\hline EUVE J1811-78.9 ........... & 2.7 & 1.5 & 0.1 & 3.3 & 4.5 & 0.3 & 5.6 & $\ldots$ & $\ldots$ & $\mathrm{dM} 5.5 \mathrm{e}$ & \\
\hline EUVE J1931-21.5 ........... & 2.9 & 1.8 & 0.2 & 5.7 & 6.4 & 0.6 & 8.8 & $\ldots$ & $\ldots$ & $\mathrm{dM} 5 \mathrm{e}$ & \\
\hline EUVE J2009-232 ............ & 0.41 & 0.02 & ... & 0.54 & 0.2 & $\ldots$ & 9.8 & $\ldots$ & $\ldots$ & K4 & \\
\hline EUVE J2056-17.1 ............ & 2.7 & 1.5 & $\ldots$ & 1.0 & 1.1 & $\ldots$ & 27.4 & 428 & 2.2 & $\mathrm{dM} 0 \mathrm{e}$ & \\
\hline EUVE J2110-19.3 ........... & 1.1 & 0.7 & 0.05 & 3.1 & 2.8 & 0.2 & 7.7 & $<50$ & $\ldots$ & dM5e & \\
\hline EUVE J2113 + 042 (b) ....... & 0.1 & $\ldots$ & $\ldots$ & 0.2 & 6.0 & $\ldots$ & $\ldots$ & $\ldots$ & $\ldots$ & K4 & Binary \\
\hline EUVE J2113 + 042 (r) ....... & 0.09 & $\ldots$ & $\ldots$ & 0.13 & $\ldots$ & $\ldots$ & $\ldots$ & $\ldots$ & $\ldots$ & & \\
\hline EUVE J $2223+25.3 \ldots \ldots \ldots \ldots$ & 0.1 & $\ldots$ & $\ldots$ & 0.12 & $\ldots$ & $\ldots$ & 7.6 & $\ldots$ & $\ldots$ & $\mathrm{dK} 7 \mathrm{e}$ & Wing em. \\
\hline EUVE J2233-09.6............ & 0.6 & 0.6 & 0.04 & 3.6 & 3.5 & 0.5 & 2.7 & $\ldots$ & $\ldots$ & $\mathrm{dM} 3.5 \mathrm{e}$ & AAO \\
\hline EUVE J2244-33.2A ......... & 4.9 & 2.5 & 0.2 & 7.3 & 7.7 & 0.5 & 10.4 & $\ldots$ & $\ldots$ & dM5e & \\
\hline EUVE J2244-33.2B ......... & 1.5 & 0.6 & 0.1 & 6.9 & 6.8 & 0.5 & $\ldots$ & $\ldots$ & $\ldots$ & $\mathrm{dM} 5 \mathrm{e}$ & \\
\hline RE J2244 + 17A .................. & $\ldots$ & $\ldots$ & $\ldots$ & $\ldots$ & $\ldots$ & $\ldots$ & 3.4 & 244 & 2.8 & K0 & \\
\hline EUVE J2329 + 41.4B ....... & 5.0 & 1.9 & $\ldots$ & 3.3 & 3.8 & 0.3 & 7.7 & 40 & $\ldots$ & $\mathrm{dM} 4.5 \mathrm{e}$ & \\
\hline EUVE J2332-012 …......... & $\ldots$ & $\ldots$ & $\ldots$ & 1.3 & $\ldots$ & $\cdots$ & 17.2 & $\ldots$ & $\ldots$ & dMle & Clouded \\
\hline
\end{tabular}

a He I 5876 A line.

b Observed EUVE Lexan/B (100 ̊) flux in units of $10^{-13} \mathrm{ergs} \mathrm{cm}^{-2} \mathrm{~s}^{-1}$ (see text).

${ }^{\mathrm{c}} \log$ of the Li $6707.8 \AA$ abundance (see text).

d Three different flux levels observed for EUVE J0419+20.9: (1) low flux level, (h) high flux level, and (f) flaring source was possibly caught flaring in 1999 September.

${ }^{\text {e }}$ For binary systems, $(b)$ indicates blue component, and $(r)$ indicates red.

${ }^{\mathrm{f}} \mathrm{H} \alpha$ wing emission.

decrease even more with increasing rotation (see Antiochos, Haisch, \& Stern 1986; Stern 1999). This suggestion, however, would predict that supersaturation will not be seen at chromospheric temperatures, a prediction that needs to be tested.

\section{NEW BINARIES AND NOTEWORTHY STARS}

Several sources showed double emission or absorption profiles, the telltale signs of spectroscopic binaries.

EUVE J0330+18.8: This source had a very curious $\mathrm{H} \alpha$ profile that resembled core emission, but after several spectra were taken at Lick and KPNO, it was resolved to be a pair of $\mathrm{F}$ stars. The $\mathrm{H} \alpha$ profile for this source is shown in Figure 7.

EUVE J2009-23.2 and EUVE J2113+04.2: These sources also showed complicated $\mathrm{H} \alpha$ emission profiles. A second spectrum of EUVE J2009-23.2 taken the following night shows two components, a broad component and a component with strong core absorption. The spectrum of EUVE J2113+04.2 showed a similar profile. We compare these $\mathrm{H} \alpha$ profiles in Figure 8. Both sources are new spectroscopic binaries.

GJ 375 and EUVE J1501-43.6: Both of these sources have strong Balmer emission, with $\mathrm{H} \alpha \mathrm{EW}$ of $\approx 2 \AA$ for the blue and red components of each star. Their $\mathrm{H} \alpha$ and $\mathrm{H} \beta$ 


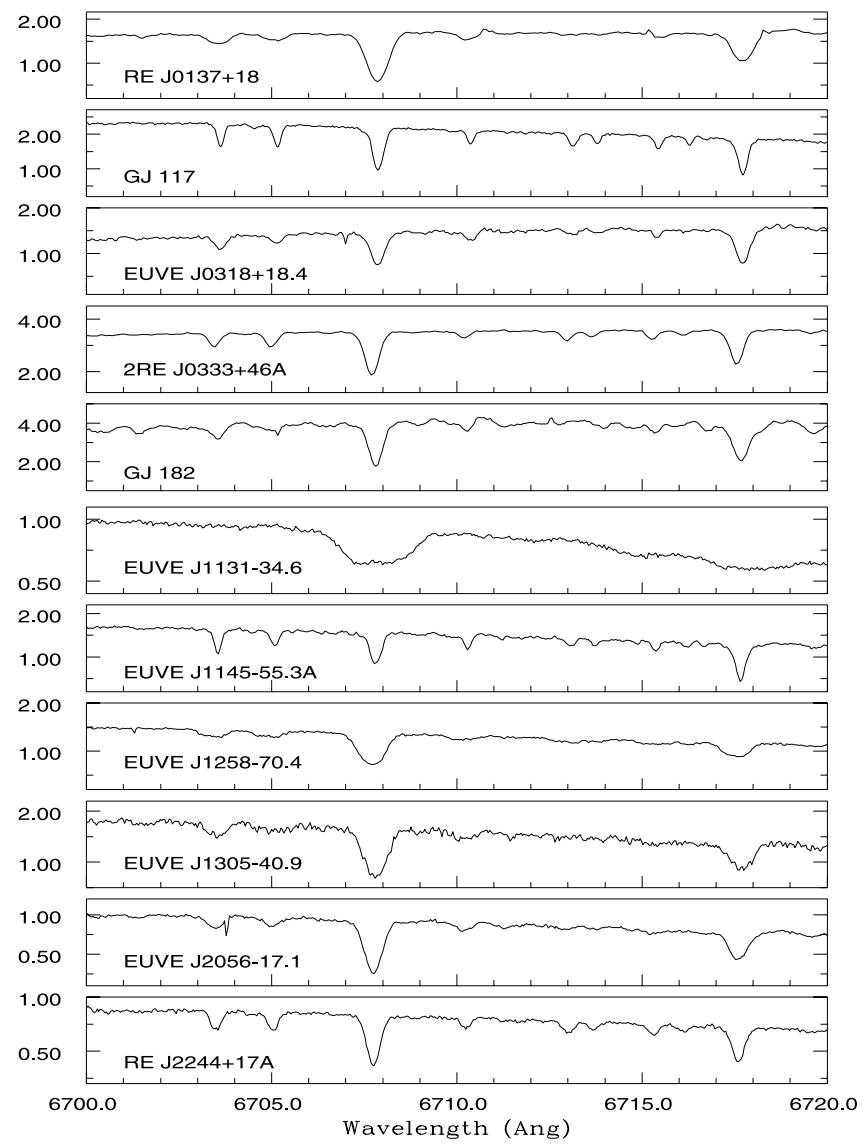
$\AA$ ).

FIG. 3.-Sample spectra of sources with lithium detections (Li I 6707.8

profiles are shown in Figure 9, along with EUVE J0825-16.3. These three sources represent new spectroscopic binaries each comprised of two dMe stars.

Emission profiles from the two components of EUVE $\mathrm{J} 1501-43.6$ are observed in $\mathrm{H} \alpha, \mathrm{H} \beta, \mathrm{He}$ I $5876 \mathrm{~A}$, and $\mathrm{Na} \mathrm{I}$.

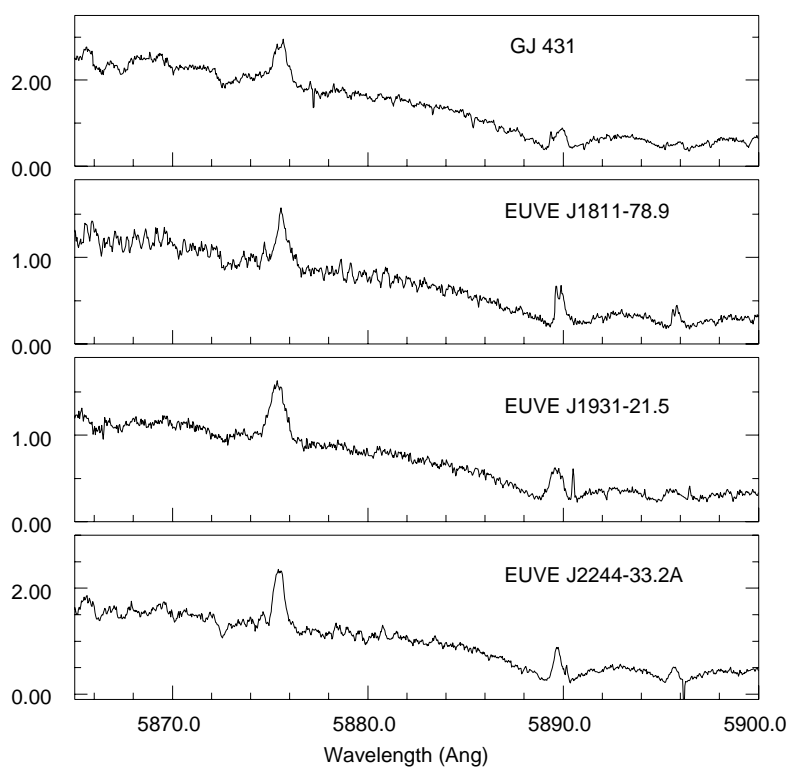

FIg. 4.-Sample He I 5876 A spectra for EUVE J1131-41.0 (GJ 431), $\mathrm{J} 1811-78.9$, J1931-21.5, and J2244-33.2A.
TABLE 3

Rotational Velocities

\begin{tabular}{|c|c|}
\hline Name & $\begin{array}{c}V_{r} \\
\left(\mathrm{~km} \mathrm{~s}^{-1}\right)\end{array}$ \\
\hline RE J0137+ 18AB ... & 15 \\
\hline EUVE J0318 + 18.4 ….................. & 10 \\
\hline 2RE J0333+46A & 8 \\
\hline EUVE J0419+21.7 ... & 25 \\
\hline EUVE J0825-16.3... & $20^{\mathrm{a}}$ \\
\hline GJ $375 \ldots$ & 10 \\
\hline EUVE J1004 + 50.3 ... & 15 \\
\hline EUVE J1131-34.6......... & 60 \\
\hline 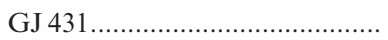 & 18 \\
\hline EUVE J1145-55.3A .................... & 5 \\
\hline 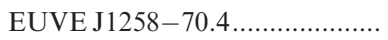 & 20 \\
\hline EUVE J1305-40.9 …...................... & 15 \\
\hline EUVE J1501-43.6 ..................... & $<5$ \\
\hline 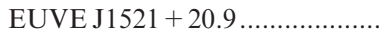 & 7 \\
\hline EUVE J1713-85.8 ... & 85 \\
\hline EUVE J1811-78.9..... & $<10$ \\
\hline EUVE J1931-21.5..... & 35 \\
\hline EUVE J2009-23.2 ... & 10 \\
\hline 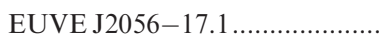 & 15 \\
\hline EUVE J2110-19.3...... & 5 \\
\hline EUVE J $2113+04.2^{\mathrm{b}}$ & 8 \\
\hline EUVE J2113 + $04.2^{\mathrm{c}} \ldots \ldots \ldots \ldots \ldots \ldots$ & 5 \\
\hline EUVE J2223 + $25.3 \ldots \ldots \ldots \ldots \ldots \ldots$ & $<5$ \\
\hline EUVE J2233-09.6 ......................... & 15 \\
\hline EUVE J2244 + 17A ........................ & 8 \\
\hline EUVE J2244-33.2A …….............. & 12 \\
\hline EUVE J2244-33.2B …................ & 22 \\
\hline
\end{tabular}

a For each member of the binary

${ }^{\mathrm{b}}$ First component.

c Second component.

Although phase coverage is limited, several spectra taken on nights 2 and 3 of the AAT run indicate that the period is approximately 1 day; however, further observations are needed for a precise determination. We point out that since both components of the binary are late $\mathrm{M}$ dwarfs, with spectral types similar to Prox Cen, this period would be consistent with the upper limit derived for the rotational velocity.

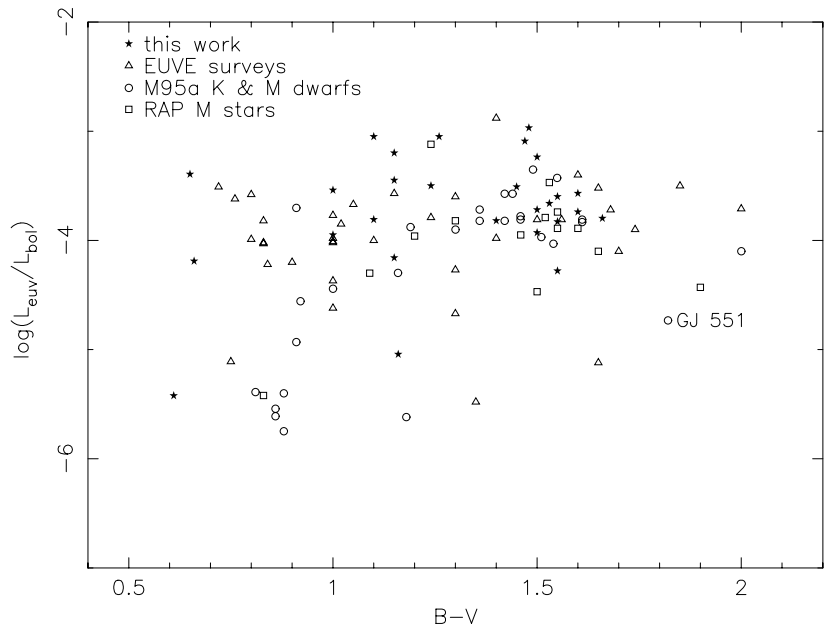

FIG. 5.- - Log of $L_{\mathrm{EUV}} / L_{\mathrm{bol}}$ plotted against $B-V$ for our sample (stars), $\mathrm{K}$ and $\mathrm{M}$ dwarfs from the EUVE all-sky survey catalogs (Bowyer et al. 1996; Lampton et al. 1997; triangles), K and M dwarfs from Mathioudakis et al. 1995b (circles), and M dwarfs from the EUVE Right Angle Program (Christian et al. 1999, squares). 


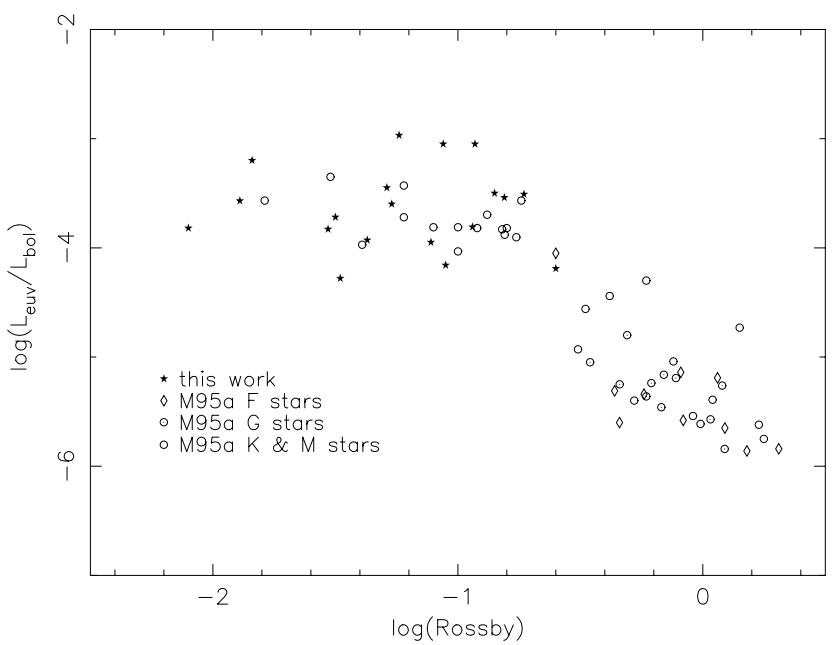

FIG. 6.-The $\log$ of the ratio of $L_{\mathrm{EUV}} / L_{\mathrm{bol}}$ plotted against the Rossby number for our sample and the F, G, K, and M dwarfs from Mathioudakis et al. (1995b). Symbols are the same as for Fig. 5 with the addition of the F stars plotted as diamonds and the $\mathrm{G}$ stars as dotted circles.

EUVE J1131-34.6: The object has the strongest $\mathrm{Li}$ line in the sample. Gregorio-Hetem et al. (1992) classify it as a T Tauri star associated with the TW Hya group. With a rotational velocity of $\approx 60 \mathrm{~km} \mathrm{~s}^{-1}$ the line profiles are dominated by rotational broadening. Strong He $5876 \AA$ emission is detected in its spectrum. A brown dwarf companion was recently identified on this source by Neuhäuser et al. (2000).

EUVE J1145-55.3: We have observed the dKe star, which is the most likely candidate of the EUV emission. Its $\mathrm{H} \alpha$ profile is double peaked. Although this could indicate that it is a binary, it is mostly likely due to strong central self-absorption caused by a high column mass in the transition region (Houdebine \& Doyle 1994).

EUVE J1258-70.4: This source is a newly identified, lithium-rich, active $\mathrm{G}$ star with $\mathrm{H} \alpha$ in emission. We have obtained a series of spectra that show variability in both the $\mathrm{H} \alpha$ profile and EW. The source could be a new RS Canum Venaticorum binary. This could explain its high $\log \left(L_{\mathrm{EUV}} /\right.$ $\left.L_{\mathrm{bol}}\right)$ of $\approx-3.4$.

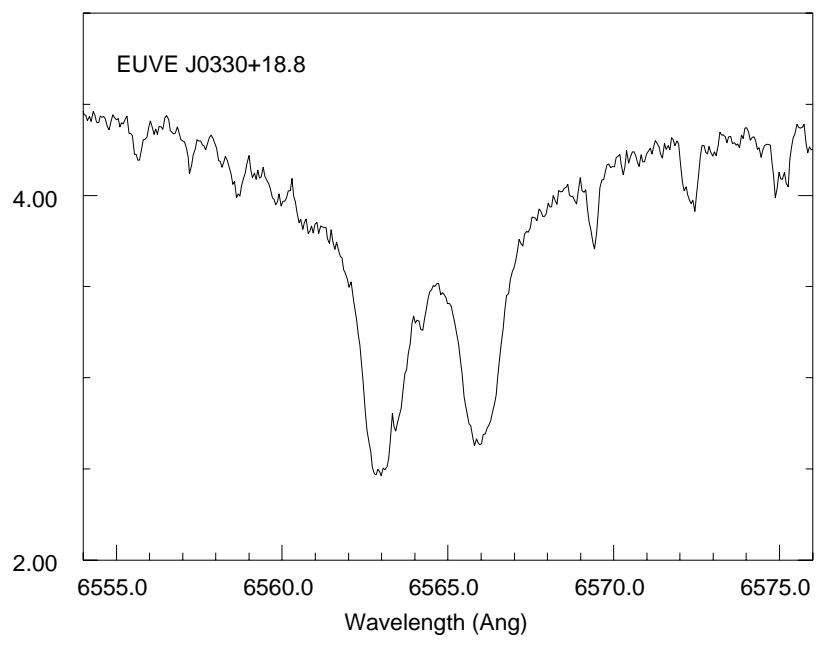

FIG. 7.- $-\mathrm{H} \alpha$ profile of EUVE J0330+18.8, a new spectroscopic binary (see text).

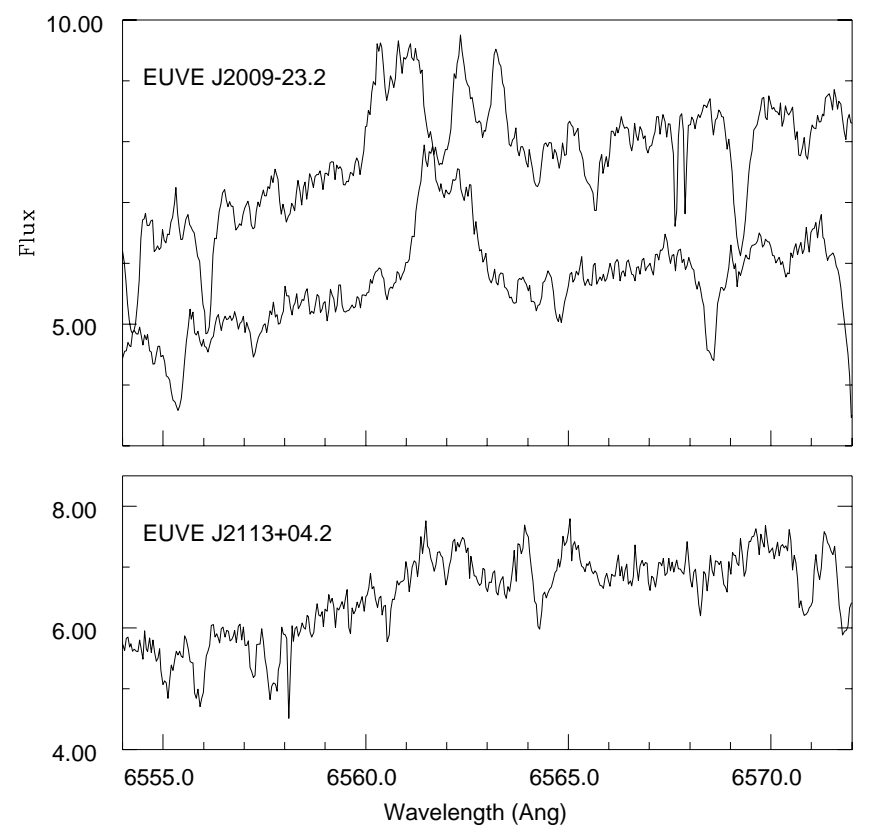

FIG. 8.-H $\alpha$ profiles for EUVE J2009-23.2 (top) and EUVE $\mathrm{J} 2113+04.2$ (bottom). The top figure shows the changing EUVE J2009-23.2 H $\alpha$ profiles from June 4 (top line) and June 5 (bottom line) indicating its binary nature (see text).

EUVE J1713-85.8: With a rotational velocity of approximately $80 \mathrm{~km} \mathrm{~s}^{-1}$, this is the fastest rotator in our sample. The $\mathrm{H} \alpha$ profile exhibits a weak absorption feature similar to the one seen on G1 890 (Byrne 1989).

EUVE J2056-17.1: This is a lithium-rich active star. Mathioudakis et al. (1995a) speculated that the high activity may contribute to Li production on the surface through spallation reactions. Van den Ancker et al. (2000) concluded that it forms a physical pair with the F8 V star HD 199143 and are both part of a nearby young $\left(\sim 10^{7} \mathrm{yr}\right)$ stellar association.

EUVE J2223+25.3: The $\mathrm{H} \alpha$ profile has a weak selfreversed feature with a central absorption below the level of the continuum. This type of profile has been predicted by model calculations. As an absorption line chromosphere progressively increases in strength, the $\mathrm{H} \alpha$ absorption profile gradually fills in, with emission peaks appearing in the wings until the line eventually goes into emission (Cram \& Mullan 1979). This source is most likely a "marginal" BY Dra star with intermediate levels of activity.

EUVE J2233-09.6: The guiding image of this source was resolved into two components at Lick in 1999 December. A spectrum was taken of the source furthest to the east. The AAT observations obtained in 2001 June 5 did not resolve the individual sources and did not show a double-line spectroscopic binary. The $\mathrm{H} \alpha$ emission measured at Lick and the AAT had similar EWs of $\approx 4 \AA$. Further observations of this source are needed to determine if it is a single-line spectroscopic binary.

\section{SUMMARY}

We have presented high-resolution optical observations of $36 \mathrm{EUV}$-selected late-type stars. Most of our sources have strong $\mathrm{H} \alpha$ emission with EWs up to $8 \AA$, while several stars show a strong Li $6707 \AA$ Aine. Rotational velocities ranged from a few to over $80 \mathrm{~km} \mathrm{~s}^{-1}$. Using these sources, the coro- 

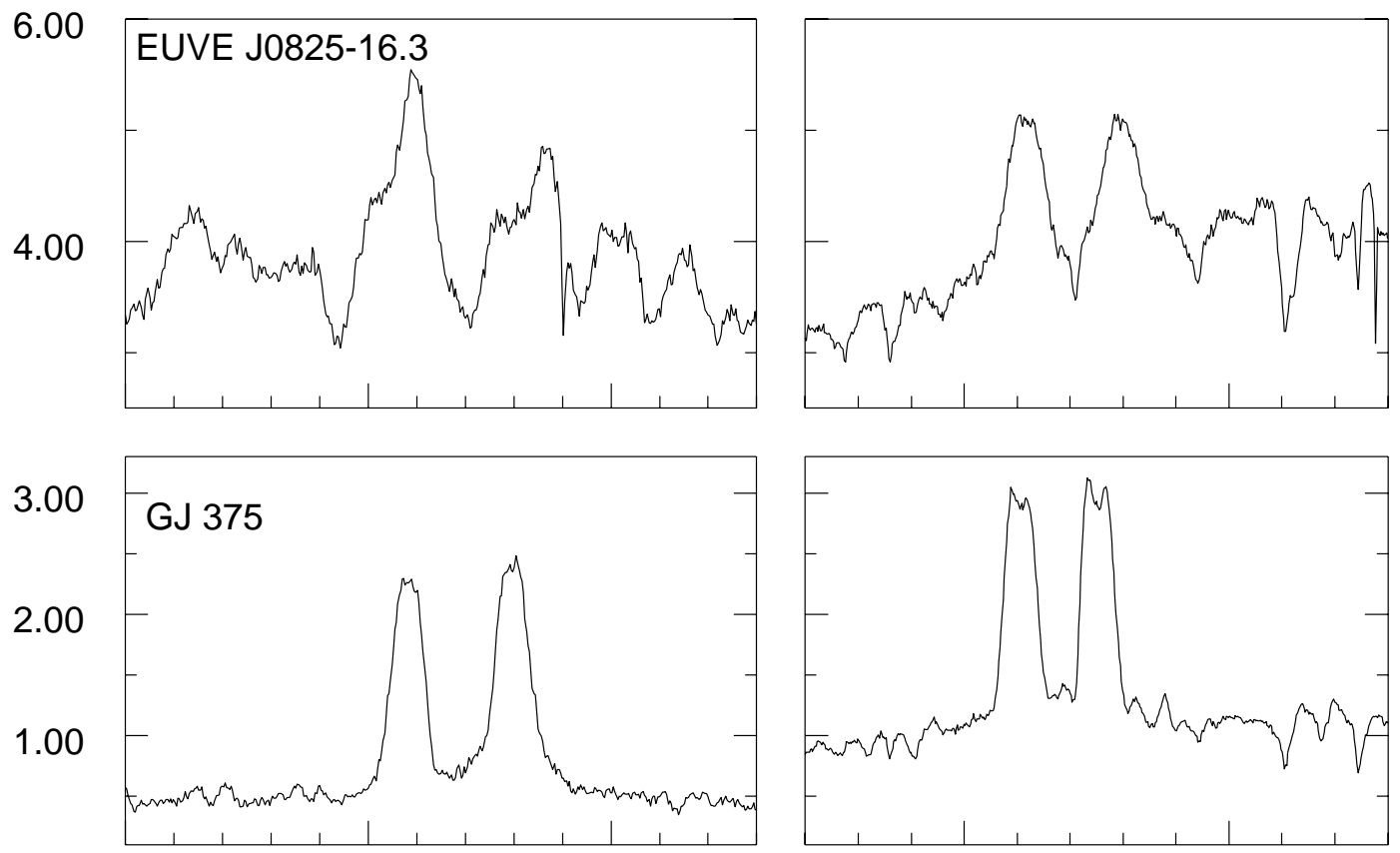

3.00

2.00
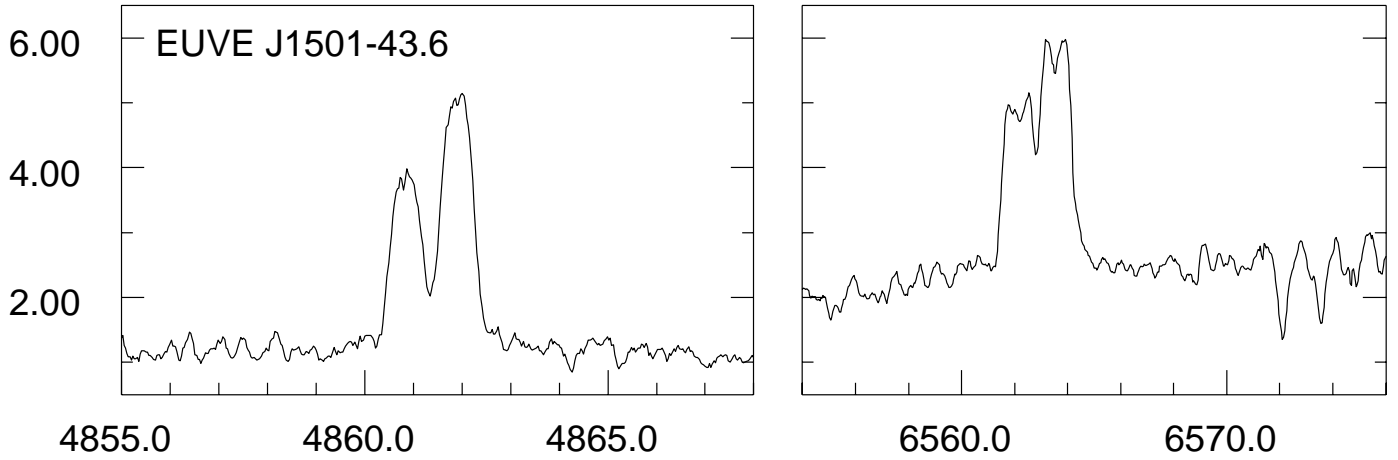

6.00

4.00

Wavelength (Ang)

FIG. 9.- $\mathrm{H} \beta$ and $\mathrm{H} \alpha$ profiles for three new spectroscopic binaries: EUVE J0825-16.3, GJ 375, and EUVE J1501-43.6 (see text)

nal saturation boundary in the EUV is now clearly defined. Our results indicate that there is a drop in the EUV emission from the corona in late $\mathrm{M}$ dwarfs possibly due to a change in the nature of the magnetic dynamo in fully convective stars. However, our sample of late M dwarfs is small, and further observations are needed, including observations of faster rotators to further explore coronal heating at small Rossby numbers $\left(\log R_{o} \approx-2\right)$. Our observations have detected seven new previously unknown spectroscopic binaries, including a pair of F stars, EUVE J0330+18.8, several late M binaries, EUVE J0825-16.3, GJ 375, EUVE J1501-43.6, EUVE J2009-23.2, EUVE J2113+04.2, and EUVE J1258-70.4, which may be a newly discovered RS CVn.
This research made use of the Kitt Peak National Observatory, National Optical Astronomy Observatory (NOAO), which is operated by the Association of Universities for Research in Astronomy, Inc., under cooperative agreement with the National Science Foundation. This work was supported by the UK Particle Physics and Astronomy Research Council. D. C. acknowledges partial support of this research with NASA Astrophysics Data Products Grants NAG 5-3470 and S-54536-G. We would like to thank T. Misch of Lick, S. Ryder of AAO, and D. Willmarth of NOAO for their excellent support during our observations. This research has made use of the SIMBAD database, operated at CDS, Strasbourg, France.
Agrawal, P. C., Rao, A. R. \& Sreekantan, V. 1986, MNRAS, 219, 225 Antiochos, S. K., Haisch, B. M., \& Stern, R. A. 1986, ApJ, 307, L55 Bowyer, S., Lampton, M., Lewis, J., Wu, X., Jelinsky, P., \& Malina, R. F. 1996, ApJS, 102, 129

Bowyer, S., \& Malina, R. F. 1991, in Extreme Ultraviolet Astronomy, ed. R. F. Malina \& S. Bowyer (New York: Pergamon), 391

Byrne, P. B. 1989, Irish Astron. J., 19, 54

Christian, D. J., Craig, N., Cahill, W., Roberts, B., \& Malina, R. F. 1999, AJ, 117, 2466

\section{REFERENCES}

Christian, D. J., Craig, N., Dupuis, J., Roberts, B. A., \& Malina, R. F. 2001, AJ, 122, 378

Craig, N., Christian, D. J., Dupuis, J., \& Roberts, B. 1997, AJ, 114, 244

Craig, N., et al. 1995, AJ, 110, 1304

Cram, L. E., \& Mullan, D. J. 1979, ApJ, 234, 579

Diego, F., Charalambous, A., Fish, A. C., \& Walker, D. D. 1990, Proc. SPIE, 1235,562

Fleming, T. A., Schmitt, J. H. M. M., \& Giampapa, M. 1995, ApJ, 450, 401 
Fruscione, A., Hawkins, I., Jelinsky, P., Wiercigroch, A. 1994, ApJS, 94, 127

Gray, D. F. 1992, The Observation and Analysis of Stellar Photospheres (2d ed.; Cambridge: Cambridge Univ. Press)

Gregorio-Hetem, J., Lepine, J. R. D., Quast, G. R., Torres, C. A. O., \& de La Reza, R. 1992, AJ, 103, 549

Haisch, B., \& Schmitt, J. H. M. M. 1996, PASP, 108, 113

Houdebine, E. R., \& Doyle, J. G. 1994, A\&A, 289, 169

Houdebine, E. R., Mathioudakis, M., Doyle, J. G., \& Foing, B. H. 1996, A\&A, 305, 209

James, D. J., Jardine, M. M., Jeffries, R. D., Randich, S., Collier-Cameron, A., \& Ferreira, M. 2000, MNRAS, 318, 1217

Jeffries, R., Bertram D. \& Spurgeon, B. R. 1995, MNRAS, 276, 397

Jeffries, R. D., \& Jewell, S. J. 1993, MNRAS, 264, 106

Küker, M., \& Rüdiger, G. 1999, A\&A, 346, 922

Lampton, M., Lieu, R., Schmitt, J. H. M. M., Bowyer, S., Voges, W., Lewis, J., \& Wu, X. 1997, ApJS, 108, 545

Linsky, J. L., \& Haisch, B. M. 1979, ApJ, 229, L27

Mathioudakis, M. 1996, Astrophys. Lett. Commun., 34, 187

Mathioudakis, M., et al. 1995a, A\&A, 302, 422

Mathioudakis, M., Fruscione, A., Drake, J. J., McDonald, K., Bowyer, S., \& Malina, R. F. 1995b, A\&A, 300, 775

Monsignori-Fossi, B., \& Landini, M. 1994, Sol. Phys., 152, 81

Neuhäuser, R., Guenther, E. W., Petr, M. G., Brandner, W., Huélamo, N., \& Alves, J. 2000, A\&A, 360, L39
Noyes, R. W., Hartmann, L. W., Baliunas, S. L., Duncan, D. K., \& Vaughan, A. H. 1984, ApJ, 279, 763

Pallavicini, R., Golub, L., Rosner, R., Vaiana, G. S., Ayres, T., \& Linsky, J. L. 1981, ApJ, 248, 279

Pavlenko, Y. V., Rebolo, R., Martin, E. L., \& Garcia Lopez, R. J. 1995, A\&A, 303, 807

Pilachowski, C., Willmarth, D., \& Goad, L. 1982, NOAO Newsl., No. 22, 7

Polomski, E., Vennes, S., Thorstensen, J. R., Mathioudakis, M., \& Falco, E. E. 1997, ApJ, 486, 179

Pye, J. P., et al. 1995, MNRAS, 274, 1165

Queloz, D., Allain, S., Mermilliod, J.-C., Bouvier, J., \& Mayor, M. 1998, A\&A, 335,183

Schmitt, J. H. M. M., Fleming, T. A., \& Giampapa, M. 1995, ApJ, 450, 392 Soderblom, D. R. 1998, Ap\&SS, 261, 59

Stauffer, J. R., Hartmann, L. W., Prosser, C. F., Randich, S., Balachandran, S., Patten, B. M., Simon, T., \& Giampapa, M. 1997, ApJ, 479, 776

Stern, R. A. 1999, in ASP Conf. Ser. 158, Solar and Stellar Activity: Similarities and Differences, ed. C. J. Butler \& J. G. Doyle (San Francisco: ASP), 47

van den Ancker, M. E., Pérez, M. R., De Winter, D., \& McCollum, B. 2000, A\&A, 363, L25

Vennes, S., Korpela, E., \& Bowyer, S. 1997, AJ, 114, 1567

Vilhu, O., \& Walter, F. M. 1987, ApJ, 321, 958

Vogt, S. 1987, PASP, 99, 1214

Wolff, S., \& Simon, T. 1997, PASP, 109, 759 